\title{
Connectivity and Coverage Preserving Schemes for Surveillance Applications in WSN
}

\author{
Lokesh Sharma \\ School of Computer Science \\ Jaypee University of \\ Informational Technology
}

\author{
Jaspreet Singh \\ School of Electronics \\ Jaypee University of \\ Informational Technology
}

\author{
Swati Agnihotri \\ School of Computer Science \\ Jaypee University of \\ Informational Technology
}

\begin{abstract}
In applications such as surveillance and target monitoring, high degree of coverage and connectivity are required. This paper investigates the problem of energy efficient coverage and connectivity for random placement of nodes such that active sensor nodes are minimized. We introduce an algorithm based on connected dominating set (CDS) and use it as a virtual backbone for network connectivity. Some nodes are refined from isolation to the backbone network, while others are connected under the tributaries of backbone network. If all the nodes are activated simultaneously, it leads to redundancy and wastage of resources in the network. In our work, coverage is achieved such that overlapping area is minimized, while connectivity of network is maintained via backbone network and its tributaries.
\end{abstract}

\section{General Terms}

Cloud computing, Grid computing, Virtualization, Parallel Computing.

\section{Keywords}

Wireless sensor networks, surveillance, backbone network, scheduling.

\section{INTRODUCTION}

Wireless Sensor Networks (WSNs) are designed by sensor nodes that communicate each other and also processing data and sensing environment wirelessly [1]. The sensor nodes are widely used in various applications these days e.g. disaster management, border protection, battlefield surveillance, space exploration, secures installation etc. Sensor nodes are deployed to operate autonomously in unattended environments in these applications [2]. The figure 1 shows architecture of battlefield surveillance where each sensor collects the data to a base station.

The networks performance can directly be affected by deployment, which is an important issue of WSNs [3]. The basic issue in WSN is the coverage problem and this problem is considered as a quality of service which can be provided by whole sensor network or by a single sensor node [4]. The main approach is to maintain the connectivity with the coverage fully preserved. The information coverage is defined according to sensor density for complete coverage [5].

In this paper, we are mainly focusing on the efficient coverage and the connectivity for randomly deployment of the sensor nodes. To maintain the network connectivity with the node connectivity, we are going to use the connecting dominating set (CDS) as a virtual backbone of the network. We should discuss about the coverage, connectivity, scheduling etc.

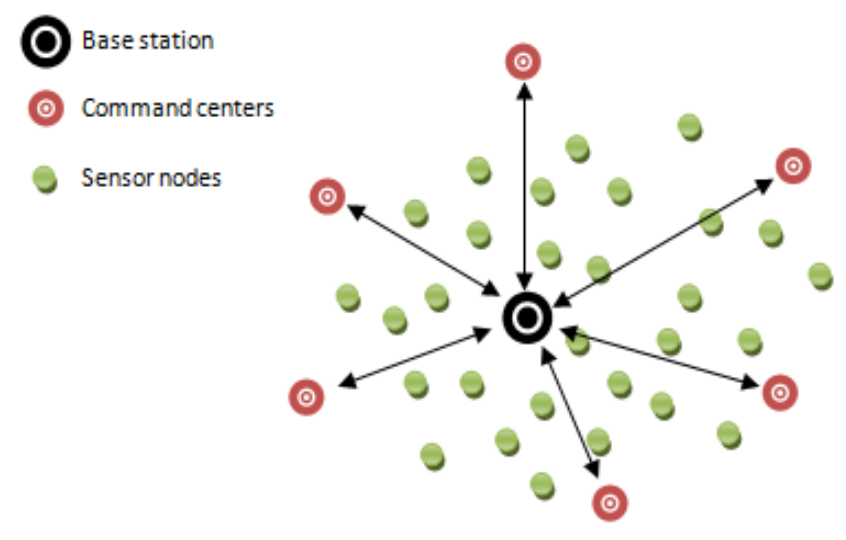

Fig. 1. A battlefield surveillance for Wireless Sensor Networks.

\subsection{Deployment}

The deployment scheme's choice is basically depends on the type of sensors, application and the environment. Nodes can be placed in an area of interest either deterministically or randomly [2]. Deployment of sensors nodes in physical unattended environment is an important issue since the performance of wireless sensor networks largely depends on deployment of sensors nodes. The deployment of sensor nodes is divided into two fractions according to the function of networks and these fractions are Coverage and Connectivity. The coverage is classified into static and dynamic category and the connectivity is divided into Pconnectivity and RAB-connectivity [3].

\section{Techniques for CDS construction}

There are different techniques by which we can design CDS, among them some are following which are efficiently applicable to WSN.

\subsubsection{Guha and Khuller's Algorithm}

There are to two Greedy Heuristic algorithm's with bounded performance guarantees. These are two strategies for building CDS.

CDS is grown from one node outward. Initially mark all nodes as white and select one white node which has maximum number of white neighbors. Mark that node as black remaining neighboring nodes as gray, now again select a node from gray nodes search for all white nodes, the one has maximum will be selected. Convert that gray node into black nodes and those white nodes into gray nodes. Iterate until all white nodes are covered.

WCDS is constructed and then intermediate nodes are selected to create CDS. First color all nodes as white. Select a node which has maximum number of white neighboring 
nodes, color that node as black remaining as gray. Again select a white node having second maximum white neighboring nodes do the same exercise. After all connect those black nodes by coloring intermediate nodes as black. Make a Steiner Tree.

1.1.2. Connectivity preserving localized coverage algorithm foe area monitoring WSN

Proposed solution Covers the area of interest (AOI), minimizing the count of active sensor nodes. If the overlap region of two sensor nodes is maximized the coverage is also maximized in region. It indicates $95 \%$ of coverage area and consuming $9.44 \mathrm{~J}$ per unit in network. There is cluster head which process the nodes location and assign each sensor a set number under which the nodes are accessible. Once set region are defined later connection between sets established. Well this algorithm is deployed over large scale sensor nodes [12].

\subsubsection{Connectivity based skeleton -Extraction in WSN}

It is totally based on geometrical environment where the sensor nodes are deployed. In this Connectivity Based Skeleton Extraction (CASE) algorithm is used to compute skeleton graph. The graphs are generated by partitioning the boundary of Sensor network identifying the skeleton points, then from those points generate skeleton arcs. By these arcs a graph is generated for WSN [13].

1.1.4. Constructing a CDS based Network backbone for energy efficiency in industrial WSN

In this we design a virtual backbone having k-way for CDS called as k- connected m-dominated (km CDS).Guha's and khuller's algorithm is implemented to design CDS. The main objective is to recover from fault tolerance; if a particular way is crushed we can go for other k-1 ways [13].

\subsection{Coverage}

The coverage problem in sensor networks is a basic issue, and considered to be a measure of the quality of service which is provided by a single sensor or by the entire network. The important aspect of coverage is to meet quality requirements, when sensors are deployed in the physical environment. If the amount of sensors deployed is less than the necessary requirement, then blind area may emerge and therefore lead to inaccurate tracking or monitoring of the area covered by the sensor network[4].The maximum coverage of some nodes in sensor networks can be described by an independent set of nodes, in which there are no intersections of sensing area between pair of nodes, an independent set of nodes is a set of nodes in graph, such that there is no link between any pair of nodes[5].The coverage issues are categorized into two parts i.e. Static and Dynamic[3].Certain tasks are performed by many WSN applications whose efficiency can be measured in terms of coverage, For these applications, it is necessary to define precise measures of coverage which impact overall system performance[6].For some WSN applications, surveillance of particular geographical areas is the main task. The coverage is basically related to the concept of area and node coverage. The parameter of area coverage is calculated by dividing the covered area to the total area of the terrain and the node coverage should be calculated by dividing number of redundant nodes to the total number of sensor nodes [7].

\subsection{Connectivity}

The key issue in sensor networks is to maintain the connectivity of the network to achieve a particular network performance. Each and every node in a network has a radio range, some nodes in a network can connect in a multi-hop manner, but not directly, increasing the radio range increase the connectivity and reduce network lifetime due to more transmission of power[3].It is must for the networks that deployment ensures that any pair of the nodes in the networks can reach each other, this is known as pure-connectivity ( $\mathrm{P}$ connectivity), which is independent of the employed routing algorithm, here power transmission is not considered and by considering power and performance, a specific routing algorithms can be used to make connection between arbitrary pair, is called routing algorithm based connectivity (RABconnectivity)[3]. In [8], it has been shown that, for strong network connectivity, it is must to achieve complete coverage of area and the range of communication is at least twice of the sensing range.

\subsection{Scheduling}

The lifetime of network can be increased by scheduling only set of sensors that are necessary to be active and other sensors must be sleeping in a network, and the better lifetime can be achieved by reducing idle listening, traffic load etc [8]. In [9], many node scheduling methods are proposed and are divided into two main categories i.e. round-based node scheduling and group-based node scheduling. According to round-based node scheduling, sensor nodes will execute the scheduling algorithm during the initialization of each round but according to group-based node scheduling, each node will execute the scheduling algorithm only once after its deployment and after the execution of scheduling algorithm, all nodes will be allocated into different groups.

A connected dominating set $\mathrm{CDS}(\mathrm{G})$ of a graph $\mathrm{G}=(\mathrm{V}, \mathrm{E})$, is defined as a subset $\mathrm{CDS}(\mathrm{G}) \subseteq \mathrm{V}(\mathrm{G})$ of $\mathrm{V}(\mathrm{G})$ such that each node in $\mathrm{V}(\mathrm{G})-\mathrm{CDS}(\mathrm{G})$ is adjacent to at least one node in $\mathrm{CDS}(\mathrm{G})$ and the produced graph (through $\mathrm{CDS}(\mathrm{G})$ ) is a connected sub graph of $G$ [10]. The nodes in sensor network communicate through multiple hops, and a connected CDS provides a backbone infrastructure [11]. In addition, backbone can be utilized for routing and activity scheduling.

\section{NETWORK MODEL}

A WSN system is represented by a graph $\mathrm{G}(\mathrm{V}, \mathrm{E})$ comprised of a set of vertices $V$ and edges $E$. The nodes $u$ and $v(u, v) \in$ $\mathrm{V}$ are said to be connected if both the nodes are within the communication range. The sensing range of node $\mathrm{v} \in \mathrm{V}$ is termed as coverage area monitored by the node.

Diagram CDS

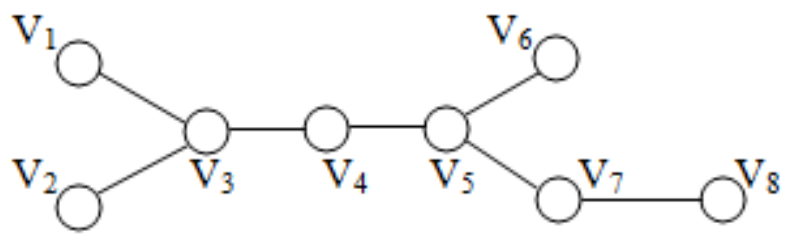

Fig.2 Graphical representation of WSN.

\subsection{Problem Statement}

\section{Construction of CDS}

The connected dominating set (CDS) of the above graph $\mathrm{G}$ shown in figure 2 is a connected sub graph of G. A CDS in figure 3 is $\left\{V_{3}, V_{4}, V_{5}, V_{7}\right\}$.

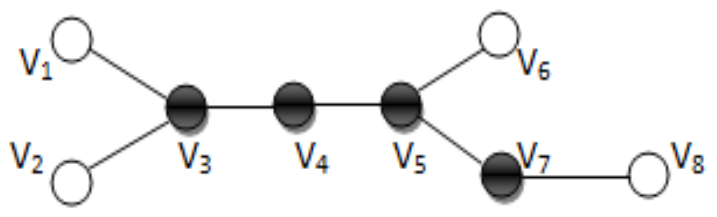

Fig.3 the connected black nodes are CDS of a graph G. 
The backbone contains $\mathrm{V}_{3}, \mathrm{~V}_{4}, \mathrm{~V}_{5}, \mathrm{~V}_{7}$ nodes only, which are shown by black colour in the figure 3 .

\subsection{Algorithm}

1. Select a network of size $X^{*} \mathrm{Y}$, where $\mathrm{X}^{*} \mathrm{Y}$ have different values $100 * 100,200 * 200$ etc.

2. Input the number of nodes and spread it over a region.

3. Select values for sensor sensing range and communication range, where communication range is twice of sensor sensing range.

4. Make sets of nodes under coverage of communication range for all the nodes call them $\mathbf{S i}$.

5. Select a node which has maximum number of nodes under his communication range call them $\mathbf{T i}$ S1, S2, S3, S4....Sensor list.

$\mathrm{T} 1, \mathrm{~T} 2, \mathrm{~T} 3, \mathrm{~T} 4$..... Target list or Required list.

6. Now take first node call it T1, select other nodes under its communication range, and select one which has maximum number of nodes and add it to Ti set.

7. Repeat UNTIL the dead end is received.

8. Connect all the nodes of Set $\mathbf{T i}$ and it works as the backbone of network which will efficiently do the communication.

The simulation was done to check out the connectivity of nodes in a different environment of $100 * 100$ and $200 * 200$ We are taking different number of nodes for these dimensions. In all the graphs, sensing range (radius) of a sensor is taken 25 every time.

\subsection{Flowchart}

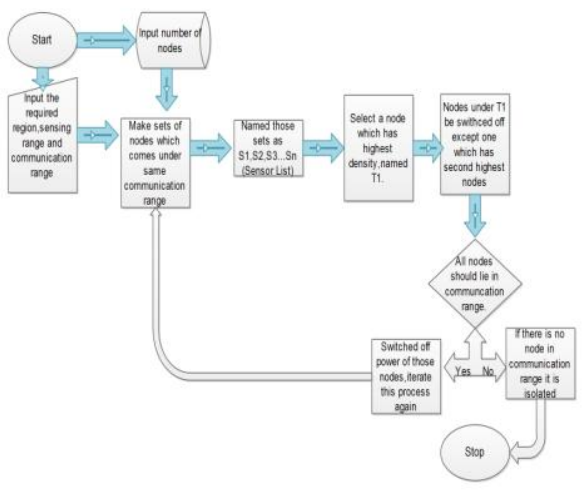

\subsection{Graphs and Table observations.}

Case 1: 25 nodes for $100 * 100$ and $200 * 200$
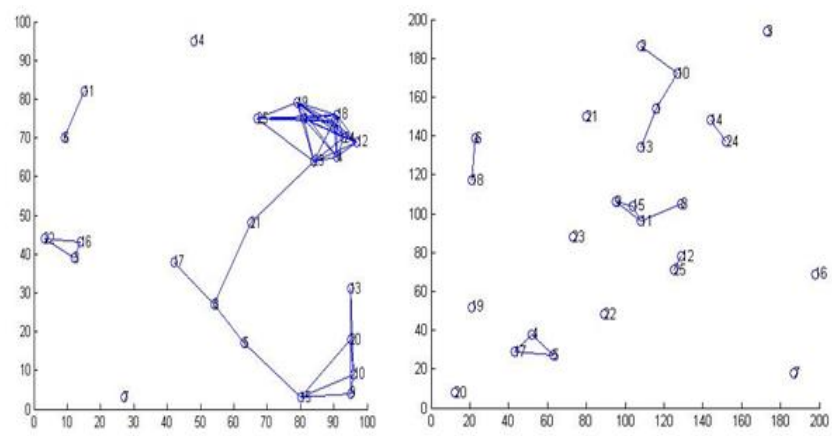

Case 2: 50 nodes for $100 * 100$ and $200 * 200$
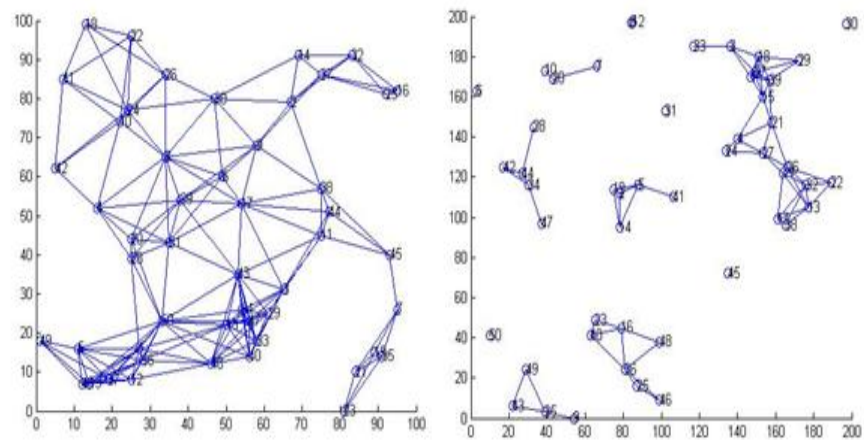

Case 3: 75 nodes for $100 * 100$ and $200 * 200$
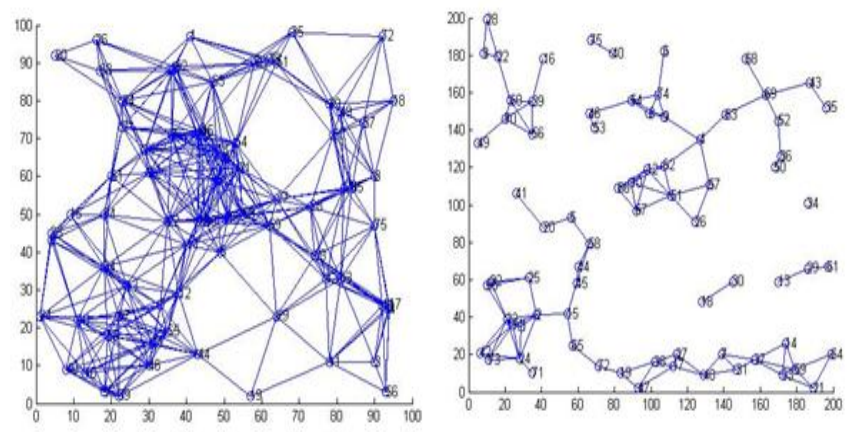

Case 4: 100 nodes for $100 * 100$ and $200 * 200$
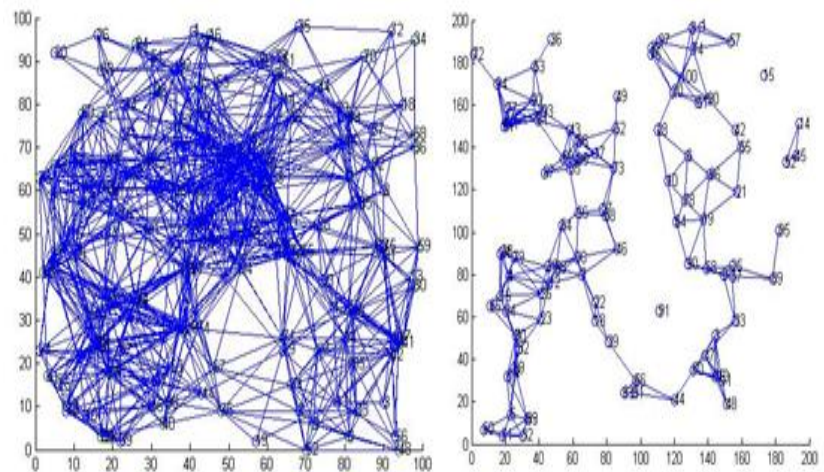


\begin{tabular}{|l|l|l|l|l|}
\hline Number of nodes & Area & $\begin{array}{l}\text { Percentage of nodes } \\
\text { in backbone }\end{array}$ & $\begin{array}{l}\text { No of nodes connected in } \\
\text { backbone (nodes in } \\
\text { backbone + nodes } \\
\text { connectivity with backbone) }\end{array}$ & $\begin{array}{l}\text { Percentage of nodes not } \\
\text { connected to backbone }\end{array}$ \\
\hline 25 & $100 * 100$ & $32 \%$ & 20 & $20 \%$ \\
\hline 50 & $100 * 100$ & $24 \%$ & 48 & $4 \%$ \\
\hline 75 & $100 * 100$ & $16 \%$ & 71 & $5.3 \%$ \\
\hline 100 & $100 * 100$ & $12 \%$ & 88 & $12 \%$ \\
\hline 25 & $200 * 200$ & $4 \%$ & 7 & $72 \%$ \\
\hline 50 & $200 * 200$ & $2 \%$ & 8 & $84 \%$ \\
\hline 75 & $200 * 200$ & $14 \%$ & 40 & $60 \%$ \\
\hline
\end{tabular}

Table 1: Observations

\section{CONCLUSION}

In this paper we try to find an efficient solution for a better WSN communication. As we have seen that our algorithm is having a superior approach towards selecting the communication region. The fascinating thing about our algorithm is that we activate a node in a sleep-wake cycle i.e. if a node is set to activate than all the other nodes are in sleep position which are in the same communication region because of this we can save the power by switching off all other nodes which are defined in the same set. Later on we are communicating between those sets. So it takes less energy to communicate, a region is defined and less number of nodes has to be in active mode. By statistical figure we can say that near about only $10 \%$ or $15 \%$ of nodes has been in active mode to make the communication efficiently. We had designed graphs by which we can conclude the regions of communication and how the data will move from one node to another node.

There is also a serious disadvantage of algorithm of isolated nodes. As there is possibility that some nodes might be in the region which is completely away from the range of Communication region, we can't establish any sort of communication between them, which is a serious impact of our algorithm.

\section{ACKNOWLEDGEMENT}

The authors would like to express their cordial thanks to $\mathrm{Mr}$. Ravindara Bhatt for his valuable advice.

\section{REFERENCES}

[1] J. You, D. Lieckfeldt, J. Salzmann and D. Timmermann, GAF \& Co : Connectivity Aware Topology Management for Sensor Networks, IEEE-2009

[2] M.Younis, K.Akkaya, Stratigies and techniques for node placement in wireless sensor networks. A Survey, Ad Hoc Networks, Vol. 6(June 2008).

[3] L.Liu, F.Xi, Z.Wang, J.Chen, Y.Sun, Deployment Issues in Wireless Sensor Networks, (C) Springer-Verlag Berlin Heidelberg 2005.

[4] Azzedine Boukerche and Xin Fei, A coveragepreserving scheme for wireless sensor network with irregular sensing range, Ad Hoc Networks Vol 5(2007).

[5] Wei An, F-M. Shao, H. Meng, The coverage-control optimization in sensor network subject to sensing area, Elsevier- 2008.

[6] A. Ghosh and S.K. Das, Coverage and connectivity issues in wireless sensor networks: A survey, Pervasive and Mobile Computing, Vol. 4, Elsevier-2008

[7] S. Oktug, A. Khalilov H. Tezcan, 3D Coverage Analysis under Heterogeneous Deployment Strategies in Wireless Sensor Networks, Fourth Advanced International Conference on Telecommunications, IEEE, 2008.

[8] Q. Zhao, M. Gurusamy, Connected K-target coverage problem in wireless sensor networks with different observation scenarios, Computer Networks Vol.52, Elsevier-2008. 
[9] L. Wang, R. Wei, Y. Lin, B. Wang, A clique base node scheduling method for wireless sensor networks, Journal of Network and Computer Applications Vol. 33, 2010.

[10] R. Misra, and C. Mandal, Minimum Connected Dominating Set Using a Collaborative Cover Heuristic for Ad Hoc Sensor Networks, IEEE Transactions on Parallel and Distributed Systems, vol. 21, no.3, March 2010.

[11] H. Raei, M. Tabibzadeh, B. Ahmadipoor, S. Saei, A Self -Stabilizing Distributed Algorithm for Minimum Connected Dominating Sets in Wireless Sensor
Networks With Different Transmission Ranges, ICACT 2009.

[12] Sudip Misra, Manikonda Pavan Kumar, Mohammad S. Obaidat: Localized coverage algorithm for area monitoring WSN, ELSEVIER, 2010.

[13] Hongbo Jiang, Chen Tian, Ying Wu: Connectivity based skeleton-Extraction in WSN, IEEE 2010.

[14] Sajid Hussain, Mubashsharul I.Shafique, Laurance T. Yang: Construction a CDS based network backbone for energy efficiency in industrial WSN,IEEE 2010 